\title{
Draft Genome Resource of a Novel Virulent Fusarium oxysporum f. sp. cubense Race 1 Strain (VCG 0124) Infecting Cavendish (AAA) Group of Banana in India
}

\author{
Raman Thangavelu, ${ }^{\dagger}$ Esack Edwin Raj, Pushpakanth P, Gopi Muthukathan, Loganathan \\ Murugan, and Subbaraya Uma \\ Indian Council of Agricultural Research National Research Centre for Banana, Tiruchirappalli \\ 620102, Tamil Nadu, India
}

\begin{abstract}
Fusarium oxysporum f. sp. cubense is one of the most destructive soilborne fungi causing Fusarium wilt disease in banana. Generally, F. oxysporum f. sp. cubense race 1 (R1) severely affects most of the banana varieties, except Cavendish banana (AAA). Here, we present the draft genome of an isolate of VCG 0124, a novel virulent R1 strain that severely affects the Cavendish group of banana isolated from the Theni district of Tamil Nadu, India. The genome assembly of R1 comprises $61,471,473$ bp with 88 contigs and 18,377 protein-coding regions. The genome contains homologs of $F$. oxysporum $\mathrm{f}$. $\mathrm{sp}$. cubense race-specific secreted-in-xylem (SIX) genes SIX1, SIX5, SIX9, and SIX13. The absence of SIX4 and SIX6 and deletion of a peptide in SIX1 virulence factor genes in the R1 (VCG 0124) strain might be the contributing factor for strains infecting Cavendish banana in India.
\end{abstract}

Fusarium oxysporum f. sp. cubense is a serious and devastating soilborne fungus causing Fusarium wilt in banana (Musa spp.) classified into F. oxysporum f. sp. cubense race 1, race 2, and race 4 based on the pathogenic specificity to host cultivars (Ploetz 2006). F. oxysporum f. sp. cubense initially invades and colonizes the banana root system and then disrupts the water translocation in vascular tissues, leading to typical wilt symptoms, which include chlorosis and necrosis of foliage, drooping of leaves, and, finally, the death of banana plants (Stover 1962). As F. oxysporum f. sp. cubense can survive in the soil for decades, banana growers are finding difficulties in growing susceptible cultivars in the field is a menacing reality. In general, banana cultivars such as Gros Michel (AAA), Silk (AAB), Pisang Awak (ABB), and Bluggoe (ABB) are susceptible to $F$. oxysporum f. sp. cubense R1, while Cavendish banana are resistant. During 2009, Thangavelu and Mustaffa (2010) noticed an unusual wilt incidence on Grand Naine (AAA) banana in the Theni district of Tamil Nadu, India and reported that a novel virulent strain of $F$. oxysporum f. sp. cubense VCG 0124 was responsible for the cause of the wilt, which is the first report of the kind in Cavendish banana. Recently, the occurrence of the strain R1 (VCG 0124) was noticed by the senior author in important banana-growing states of India (namely, Gujarat, Maharashtra, Madhya Pradesh, and West Bengal), where it is attacking banana crops grown in more than 1,000,000 ha (personnel communication). In this context, a study was initiated to understand the genome of an $F$. oxysporum f. sp. cubense virulent strain in detail in comparison with the reference F. oxysporum f. sp. Iycopersici 4287 (GCA_000149955.2) and R1 (GCA_000350345.1). Here, we report the draft genome of the F. oxysporum f. sp. cubense VCG 0124 and discuss

\section{Funding}

Financial support for this study was provided through the extramural project rendered by the Indian Council of Agricultural Research (ICAR), Ministry of Agriculture \& Farmers' Welfare, Government of India, New Delhi, ICAR grant 7 (27)2016/HS-1.

\section{Keywords}

Cavendish banana, Foc R1, Fusarium oxysporum f. sp. cubense $\mathrm{R} 1$, Fusarium wilt, secreted in xylem, virulence-associated genes, whole-genome sequencing

\footnotetext{
The author(s) declare no conflict of interest.

Accepted for publication 11 March 2021.

() 2021 The American Phytopathological Society

${ }^{\dagger}$ Corresponding author: R. Thangavelu; rtbanana@gmail.com

R. Thangavelu and E. Edwin Raj contributed equally to this work. 
Table 1. Summary of genome characteristics and predicted features of reference and assembled genomes of Fusarium oxysporum f. sp. lycopersici 4287 and F. oxysporum f. sp. cubense race 1 (R1)

\begin{tabular}{lccc} 
& \multicolumn{3}{c}{ Genome assembly numbers (strain) } \\
\cline { 2 - 4 } Characteristics & GCA_000149955.2 & GCA_000350345.1 & GCA_011316005.3 \\
(R1)
\end{tabular}

${ }^{a}$ Genome assembly numbers of available in the NCBI database.

the secreted-in-xylem (SIX) genes of the isolate and the possible reason for the infection on Cavendish banana.

A detailed description of the origin and isolation of the F. oxysporum f. sp. cubense strain, confirmation using nit-M testers of known vegetative compatibility groups, volatile odor production, and its pathogenicity were furnished by Thangavelu and Mustaffa (2010). The genomic DNA was isolated from the single spore culture of late-log-phase growth (grown in the potato dextrose broth medium at $25 \pm 2^{\circ} \mathrm{C}$ without shaking) by the cetyltrimethylammonium bromide method (Doyle and Doyle 1987) followed by proteinase $\mathrm{K}$ and RNase digestion (Roche Diagnostics). DNA purity was measured by the A260/A280 ratio using NanoDrop ND-1000 (Nanodrop) and agarose gel electrophoresis. The genomic library was prepared from a sheared DNA fraction of approximately $300 \mathrm{bp}$ using Illumina paired-end TruSeq DNA sample preparation kits according to the manufacturer's instructions. Fungal libraries were sequenced using the Illumina NextSeq 500 system (M/s. Genotypic) for $150 \times 2$ cycles, generating approximately 2.6 million paired-ends reads amounting to approximately 17.16 million total raw reads. The short-read sequences of R1 were assembled using MaSuRCA v.3.2.4 (Zimin et al. 2013), mapped with F. oxysporum f. sp. lycopersici 4287 using BWA v.0.7.12 (Li and Durbin 2009), and annotated using Fungal Ensemble gene annotation system (https://fungi.ensembl.org/index.html). The draft genome sequence of R1 VCG 0124 was $61.47 \mathrm{Mb}$ in length with $48.4 \% \mathrm{GC}$ content (Table 1). The genome consisted of $88 \mathrm{con}$ tigs of 900 to $6,854,980 \mathrm{bp}$, with an average contig size of $698,539 \mathrm{bp}$. The completeness was estimated using BUSCO (v.3.0.2) with the Sordariomycetes data set (Waterhouse et al. 2018 ) and resulted in $96.5 \%(3,680)$ which occurred as intact and single copies out of 3,817 target genes. This is in accordance with Asai et al. (2019) and Warmington et al. (2019), who reported the whole-genome sequences of $F$. oxysporum f. sp. cubense using BUSCO. Functional annotation of AUGUSTUS (v.3.3)-predicted (Stanke et al. 2006) protein-coding genes was performed by combining InterProScan (Jones et al. 2014) and BLASTp searches against the UniProtKB Fungi protein database (ConsortiumUniProt 2018). In total, 18,370 genes were annotated from 18,377 protein-coding genes using the UniProt database with a cutoff E-value of $10^{-5}$ (Boutet et al. 2007). Based on the similarity search with the cluster of orthologous grouping (COG) database (https://www.ncbi.nlm.nih.gov/COG/), COG of the proteins was done with an E-value threshold of $10^{-5}$ (Conesa et al. 2005). Of the annotated proteins, 2,426 (13.2\%) were associated with biological processes, 7,241 (39.4\%) were associated with cellular processes and signaling, and 8,674 (47.2\%) were associated with molecular functions. However, $37(0.2 \%)$ protein-coding genes were not categorized into any of the COG classes and, thus, were considered to be proteins of uncharacterized functions or features. A further comparison with the reference R1 (GCA_000350345.1) revealed that there were 326 unaligned protein-coding sequences present in the studied genome, which is estimated to be approximately $3.7 \mathrm{Mb}$ in size. Similar comparison with $F$. oxysporum II5 (GCA_000260195.2) reveled that there were 495 unaligned protein-coding sequences recognized in this genome. which is about $5.2 \mathrm{Mb}$ in size. These are considered as unique regions present only in the genome of the study, which differentiates them from the reference 
F. oxysporum f. sp. cubense R1 and R4 strains based on genome organization. To visualize the localization of blocks of collinearity, rearrangements, and inversions, MAUVE program v20150226 was used with a weight close to 5,000 (Darling et al. 2004). The results showed that there were $900(4,60,874 \mathrm{bp})$ and 1,135 (5,20,324 bp) homologous regions of locally collinear blocks conserved in the genome of $F$. oxysporum f. sp. lycopersici 4287 and $F$. oxysporum II5, respectively, with R1.

Protein coding genes predicted in this study and subjected to Plant Host Interaction search (http://www.phi-base.org) showed that there were 1,042 genes in the genome of $F$. oxysporum f. sp. cubense R1 (VCG 0124), in which 30 are unique in comparison with reference R1. To characterize the isolate by the presence and absence of SIX protein genes (SIX1 to SIX14), BLASTX analysis was performed. Among the SIX genes, SIX1, SIX5, SIX9, and SIX13 were commonly found to present in both the reference ( $F$. oxysporum f. sp. lycopersici 4287 and $F$. oxysporum f. sp. cubense R1) and R1 (VCG 0124) genomes, whereas SIX4 and SIX6 were absent in the genome of R1 (VCG 0124) infecting Cavendish banana when compared with reference $F$. oxysporum f. sp. cubense R1 and $F$. oxysporum f. sp. lycopersici 4287. Two homologous copies of SIX1 and SIX9 were present in chromosome 14 and $S I X 13$ in chromosome 6, whereas a single copy of SIX5 was noticed in chromosome 14. The contig CM027182.1 of chromosome 14 between 2,04,004 and 2,04,365 nucleotides encoded a SIX1 gene (alternatively, AVR3) has a sequence polymorphism at amino acid position 205 to 214 (by deletion). This is in corroboration with the report of Li et al. (2016), who found a deletion in the SIX1 gene in F. oxysporum f. sp. conglutinans that enhanced the virulence in infecting cabbage. They also reported that the modification in SIX1 is required for the compatible interaction with the other host cultivars. Further comparison of $\mathrm{R} 1$ with the genome of other $F$. oxysporum f. sp. cubense races may provide insights into why the strain is also attacking Cavendish banana, which will be explored in our future studies.

Nucleotide sequence accession numbers. The assembled short-read genome sequences of F. oxysporum f. sp. cubense race 1 (VCG 0124) infecting Cavendish banana has been deposited at DNA Data Bank of Japan/European Nucleotide Archive/GenBank under accession number GCA_011316005.3 (PRJNA552447), which is the third version.

\section{Acknowledgments}

We thank the Director, Indian Council of Agricultural Research National Research Centre for Banana, Tiruchirappalli for the facilities provided.

\section{Literature Cited}

Asai, S., Ayukawa, Y., Gan, P., Masuda, S., Komatsu, K., Shirasu, K., and Arie, T. 2019. High-quality draft genome sequence of Fusarium oxysporum f. sp. cubense strain 160527, a causal agent of Panama disease. Microbiol. Resour. Announce. 8:e00654-19.

Boutet, E., Lieberherr, D., Tognolli, M., Schneider, M., and Bairoch, A. 2007. UniProtKB/Swiss-Prot. Pages 89-112 in: Plant Bioinformatics. Methods in Molecular Biology, vol. 406. D. Edwards, ed. Humana Press, Totowa, NJ, U.S.A.

Conesa, A., Götz, S., García-Gómez, J. M., Terol, J., Talón, M., and Robles, M. 2005. Blast2GO: A universal tool for annotation, visualization and analysis in functional genomics research. Bioinformatics 21:3674-3676.

ConsortiumUniProt. 2018. UniProt: The universal protein knowledgebase. Nucleic Acids Res. 46:2699.

Darling, A. C. E., Mau, B., Blattner, F. R., and Perna, N. T. 2004. Mauve: Multiple alignment of conserved genomic sequence with rearrangements. Genome Res. 14:1394-1403.

Doyle, J. J., and Doyle, J. L. 1987. A rapid DNA isolation procedure for small quantities of fresh leaf tissue. Phytochem. Bull. 19:11-15.

Jones, P., Binns, D., Chang, H. Y., Fraser, M., Li, W., and McAnulla, C. 2014. InterProScan 5: Genome-scale protein function classification. Bioinformatics 30:1236-1240.

Li, H., and Durbin, R. 2009. Fast and accurate short read alignment with Burrows-Wheeler transform. Bioinformatics 25:1754-1760.
Li, Z. F., He, C. L., Wang, Y., Li, M. J., Dai, Y. J., and Wang, T. 2016. Enhancement of trichothecene mycotoxins of Fusarium oxysporum by ferulic acid aggravates oxidative damage in Rehmannia glutinosa Libosch. Sci. Rep. 6:33962.

Ploetz, R. C. 2006. Fusarium wilt of banana is caused by several pathogens referred to as Fusarium oxysporum f. sp. cubense. Phytopathology 96:653-656.

Stanke, M., Keller, O., Gunduz, I., Hayes, A., Waack, S., and Morgenstern, B. 2006. AUGUSTUS: Ab initio prediction of alternative transcripts. Nucleic Acids Res. 34:W435-W439.

Stover, R. H. 1962. Fusarial Wilt (Panama Disease) of Bananas and Other Musa Species. Commonwealth Mycological Institute, United Kingdom.

Thangavelu, R., and Mustaffa, M. M. 2010. First report on the occurrence of a virulent strain of Fusarium wilt pathogen (race-1) Infecting Cavendish (AAA) group of bananas in India. Plant Dis. 94:1379.

Warmington, R. J., Kay, W., Jeffries, A., O'Neill, P., Farbos, A., Moore, K., Bebber, D. P., and Studholme, D. J. 2019. High-quality draft genome sequence of the causal agent of the current Panama disease epidemic. Microbiol. Resour. Announce. 8:e00904-19.

Waterhouse, R. M., Seppey, M., Simao, F. A., Manni, M., loannidis, P., and Klioutchnikov, G. 2018. BUSCO applications from quality assessments to gene prediction and phylogenomics. Mol. Biol. Evol. 35:543-548.

Zimin, A. V., Marçais, G., Puiu, D., Roberts, M., Salzberg, S. L., and Yorke, J. A. 2013. The MaSuRCA genome assembler. Bioinformatics 29:2669-2677. 